\title{
Presentación \\ Diálogos Chile-Francia: discursos mediáticos, su producción y su circulación
}

De muy larga data, las relaciones entre Chile y Francia en el campo de las ciencias sociales, las artes y las humanidades, han tenido una importante influencia en el pensamiento teórico y en las prácticas científicas y profesionales de los intelectuales chilenos. Imposible pasar por alto la vigencia que aún conservan en la discusión disciplinaria pensadores como Bourdieu, Morin, Ricoeur, Foucault, Levi-Strauss, Lacan, Benveniste o Barthes, sólo por nombrar algunos ejemplos notables.

Esta presencia del pensamiento francés en Chile ha contribuido de manera significativa a ampliar y diversificar el abanico de perspectivas epistemológicas desde donde se intentan comprender los fenómenos sociales, enriqueciendo el panorama de la reflexión crítica al interior de universidades y organizaciones sociales y políticas de nuestro país.

En el nivel específico de la labor universitaria, esta relación se ha ido consolidando con la creación de instancias de docencia, investigación y difusión tales como la Cátedra Pablo Neruda (IAEHAL-Universidad de París III), el Colegio Doctoral Franco-Chileno, la Cátedra Michel Foucault (Universidad de Chile), el programa ECOS-SUD (que en Chile gestiona y coordina CONICYT), entre otros. Este proceso de institucionalización deloslazos académicos en el ámbito de las ciencias sociales y las humanidades, ha tenido un efecto muy importante en la construcción de vínculos simétricos entre intelectuales franceses y chilenos, poniendo en valor y difundiendo su producción científica común, lo que representa un índice de la madurez alcanzada en estos años.

Sin embargo, hay un ámbito respecto del cual aún persiste un importante déficit en el plano de la discusión e intercambio entre Chile y Francia: este es el de las ciencias de la comunicación y, en particular, el referido a la producción teórica en torno del fenómeno de los medios de comunicación.

Dominada por dos tradiciones fuertemente opuestas, la investigación sobre medios de comunicación en Chile ( $y$, en general, en Latinoamérica) se ha movilizado, principalmente, entre la sociología norteamericana de orientación funcionalista y la teoría neo-crítica inspirada en la Escuela de Frankfurt. Cada una en espacios simbólicos y profesionales distintos, constituyeron comunidades que, más allá de sus notorias diferencias y escasos vínculos, han sido decisivas para la instalación y desarrollo del campo en nuestro país.

Por su parte, el temprano desarrollo de los estudios mediáticos en Francia ha estado dominado, desde su origen, por marcos conceptuales que surgen de la lingüística, la teoría de la enunciación y la teoría literaria. Esta singularidad epistemológica de la investigación francesa sobre los medios de comunicación le ha permitido construir una fuerte identidad en la escena 
internacional, estableciendo una importante diferencia de enfoque respecto de la tradición anglosajona y latinoamericana.

Sólo a partir de encuentros esporádicos y puntuales, estas dos lógicas de trabajo no han tenido muchas oportunidades de poner en discusión sus fundamentos teóricos y sus prácticas metodológicas, perdiendo una interesante oportunidad para la reflexión comparada y conjunta que permita enriquecer la mirada.

\section{Un primer intento}

Esta publicación es el resultado de la labor conjunta de un grupo de investigación chilenofrancés que, motivado por las dificultades y desafíos que impone el trabajo intercultural, se ha interesado en abrir un espacio de diálogo y discusión que trascienda las fronteras nacionales, disciplinarias y epistemológicas respecto de la investigación sobre los medios de comunicación.

Con el objetivo de poner de relieve las lógicas que sustentan el quehacer de las comunidades académicas de uno y otro país, hemos estructurado el presente diálogo a partir de dos volúmenes que intentan abarcar parte de la diversidad y complejidad de las manifestaciones e intereses respecto del fenómeno mediático: desde los procesos materiales y simbólicos de producción y circulación de los discursos, hasta las prácticas de mediación, consumo, recepción o apropiación que se desprenden de los usos que los sujetos socialmente inscritos dan a los medios de comunicación, sus mensajes y representaciones.

El presente número de la revista Comunicación y Medios se concentra en la problemática de la producción y circulación de los discursos mediáticos, intentando poner en evidencia las divergencias y convergencias entre los dos campos "científico-culturales". En este sentido, queremos proponer una lectura de segundo orden al conjunto de artículos de la monografía que, junto con apreciar el aporte específico de cada texto, permita también preguntarse sobre el posible carácter indicial de las temáticas, perspectivas, objetos y métodos elegidos por los autores de uno y otro país.

De este modo, la pregunta sobre las formas que adquiere la representación de lo popular en el cine chileno contemporáneo, en el artículo de Eduardo Santa Cruz, pone de manifiesto un interés por las contradicciones, explícitas e implícitas, que se hacen visibles a través de los discursos sobre la identidad nacional, siendo una oportunidad para reconocer el estado actual de las hegemonías ideológicas, según el autor. En una clave muy próxima a la anterior, el artículo de Gustavo González se propone hacer evidente, a partir de la constatación de la isotopía del "antisocial" en los discursos mediáticos dominantes, las estrategias de criminalización y estigmatización que opera el poder frente a la movilización y crítica social. Ambas perspectivas podrían ser constitutivas de una constante muy bien representada dentro de las preocupaciones de los intelectuales chilenos interesados en la comunicación mediática. 
Por su parte, el artículo de Oscar Garaycochea, nos propone una reflexión lógica respecto de los productos de la industria cultural del cine, despegándose de las perspectivas éticas y estéticas propias del análisis audiovisual tradicional. De este modo, el autor pone en juego una mirada analítica respecto de los procedimientos y lógicas discursivas polares de enmascaramiento y ostensión de la propia fuente enunciativa, pero sin abandonar la relación de estas prácticas con las formas de producción concretas del cine como industria, lo que nos reenvía, probablemente, a otro rasgo identitario del análisis mediático latinoamericano que intenta contextualizar los fenómenos particulares dentro de explicaciones globales de lo social.

Por último, el artículo de Enrique Vergara y Maite Rodríguez nos propone una lectura inductiva del fenómeno mediático, poniendo en obra un movimiento lógico muy diferente a los anteriores. A partir de los resultados de una investigación empírica sobre un caso específico, los autores abordan el estado actual y los desafíos teóricos y prácticos de la publicidad, avanzando la necesidad de repensar esta actividad significante.

Por su parte, los cinco autores franceses presentes en este número, si bien confluyen en su interés común por los fenómenos mediáticos, se distinguen por su anclajes teóricos y disciplinarios y por los "modos de entrada" al análisis de los objetos.

Inspirado por los trabajos de Pierre Bourdieu y por la teoría de los campos, el sociólogo Dominique Marchetti pone su mirada sobre el polo de la producción, interrogándose sobre las dificultades de orden metodológico y epistemológico que plantea este tipo de aproximaciones. Élda pistas para comprender cuálesson las lógicas de producción de la información periodística, insistiendo sobre un doble imperativo: poner atención en las lógicas relacionales presentes al interior del campo periodístico, sin dejar de interesarse por las relaciones entre éste y otros campos (jurídico, político, económico). También sociólogo de formación, Rémy Rieffel ha desarrollado numerosas investigaciones sobre el medio profesional utilizando otros métodos y apoyándose en otras fuentes teóricas. Inspirándose a la vez en Max Weber y Norbert Elias, sugiere articular los enfoques relacional y comprensivo. Propone la necesidad de abordar las condiciones de producción de los periodistas y de otros actores de la información sin disociar éstas de la cuestión de los soportes de la información, es decir, de sus identidades discursivas y de los modos de apropiación por parte de los lectores. Psicólogos del lenguaje, Didier Courbet y Marie-Pierre Fourquet dan cuenta en su artículo de los resultados de una investigación sobre creadores de la publicidad en Internet. Situados desde otro paradigma presente en Francia en las ciencias de la comunicación, ellos se interesan por los procesos psicológicos puestos en juego por los creadores publicitarios para construir mensajes persuasivos, destacando el rol determinante de sus "teorías implícitas" sobre la influencia de la publicidad y mostrando que este tipo de creación mediática da lugar a un proceso de racionalización de las elecciones operadas, determinados por las demandas del anunciador.

Béatrice Fleury-Vilatte y Jacques Walter, en cambio, se sitúan, a través del análisis metódico de un corpus de prensa, en el lugar desde el cual se construye el discurso mediático. 
Ellos consideran, sin embargo, que la comprensión de los mecanismos puestos en obra en este nivel, necesita tomar en cuenta los dos otros "lugares de pertinencia" distinguidos por Patrick Charaudeau: las condiciones de producción y las modalidades de interpretación en la recepción. Utilizando el ejemplo del proceso judicial a Maurice Papon (un ex alto funcionario acusado de haber colaborado en la deportación de judíos en la segunda guerra mundial), de fuerte impacto en Francia, ellos muestran cómo se produjo en esta ocasión la mediatización del discurso experto de los historiadores y cómo esto se tradujo en una controversia más amplia sobre el sitio de la Historia en la escena de lo Político.

Distanciándose de teorizaciones marcadas por ciertas formas de "mediacentrismo", Jocelyne Arquembourg, cuyo trabajo también se apoya en el análisis metódico de un corpus, muestra en este estudio que la temporalización de un acontecimiento en vivo y en directo obedece a orientaciones y perspectivas distintas a las del acontecimiento en diferido. Inspirada por el análisis praxeológico del espacio público de Louis Quéré, produce resultados originales integrando marcos conceptuales ya presentes en el campo de reflexión, muy activos en Francia y en Bélgica (la escuela de Louvain-la-Neuve) sobre los relatos mediáticos. Ella profundiza en su artículo esta línea de trabajo analizando los relatos televisivos franceses sobre la "cuestión irakí, mostrando cómo las producciones mediáticas no rinden cuenta de lo real sino que construyen horizontes de expectativa para los públicos, así como matrices de interpretación y de juicio sobre la actualidad.

De esta manera, queremos presentar a los lectores un primer intento de diálogo entre la investigación mediática de Chile y Francia, el cual esperamos, pueda fortalecer una perspectiva científica intercultural que permita ir superando la tendencia hacia la universalización, muchas veces inapropiada, de marcos teóricos, herramientas conceptuales y temáticas que deberían ponerse a prueba en contextos sociales y culturales distintos.

Guy Lochard

Universidad de París III
Bernardo Amigo

Universidad de Chile 